\title{
Expression profiles of 507 proteins from a biotin label-based antibody array in human colorectal cancer
}

\author{
HISAAKI MIYOSHI $^{1}$, ASAHIRO MORISHITA ${ }^{1}$, JOJI TANI ${ }^{1}$, TEPPEI SAKAMOTO ${ }^{1}$, \\ KOJI FUJITA ${ }^{1}$, AKIKO KATSURA ${ }^{1}$, MIWA TATSUTA ${ }^{1}$, TAKAKO NOMURA ${ }^{1}$, HIROHITO YONEYAMA ${ }^{1}$, \\ HISAKAZU IWAMA ${ }^{2}$, YASUYUKI SUZUKI ${ }^{3}$ and TSUTOMU MASAKI ${ }^{1}$ \\ ${ }^{1}$ Department of Gastroenterology and Neurology, ${ }^{2}$ Life Science Research Center, \\ ${ }^{3}$ Department of Gastroenterological Surgery, Kagawa University Faculty of Medicine, \\ Kita-gun, Kagawa 761-0793, Japan
}

Received June 30, 2013; Accepted August 29, 2013

DOI: 10.3892/or.2013.2935

\begin{abstract}
Molecular-targeted therapy is one of the most promising therapies for patients with advanced-stage colorectal cancer (CRC). However, a wide range of proteins have unknown expression levels in CRC. The purpose of the present study was to determine the expression levels of various proteins related to colorectal carcinogenesis and cancer development. We examined the expression levels of 507 target proteins using a biotin label-based antibody array in 6 human CRC tissues. We also analyzed the clinicopathological features of CRC patients. In CRC tissues, IL-1 $\alpha$, GRO, Glut5, MIG, ICAM-5, VE-cadherin, uPA and Leptin R were increased when compared to levels in normal colon tissues. MPIF-1/CCL23, FGF R5, MIP2, SAA and IL-18 R $\beta$ were strongly upregulated in rectal cancer when compared to the levels in non-rectal cancer. These data suggest that differential protein expression profiles exist under different conditions, including carcinogenesis and CRC localization. Therefore, an exhaustive analysis of protein expression levels using a biotin label-based antibody protein array is a potentially useful tool for identifying novel individual therapies for CRC patients.
\end{abstract}

\section{Introduction}

Colorectal cancer (CRC) is the fourth leading cause of cancerrelated death in the world (1). Epidemiologically, a Western diet, alcohol, smoking and obesity are related to an increased risk of CRC and mortality $(2,3)$. Although screening and prevention efforts have been promoted, a small fraction of

Correspondence to: Professor Tsutomu Masaki, Department of Gastroenterology and Neurology, Kagawa University Faculty of Medicine, 1750-1 Ikenobe, Miki-cho, Kita-gun, Kagawa 761-0793, Japan

E-mail: tmasaki@med.kagawa-u.ac.jp

Key words: colorectal cancer, biotin label-based antibody array, hierarchical clustering analysis
CRC cases are still discovered as unresectable tumors due to metastasis to critical organs (4). Radiation therapy (5) and chemotherapy (6) have major limitations to the treatment of CRC patients. Therefore, a novel therapeutic approach is required for CRC.

Antibody arrays represent a promising technology for quantitative and exhaustive protein profiling (7). Sandwichbased arrays are frequently used due to their high sensitivity, specificity and cost effectiveness. However, a pair of antibodies could cross-react and hamper the appropriate reaction with proteins in the array. Current antibody protein arrays also have limited abilities to provide a broad, panoramic view of protein expression levels. Recently, a new antibody array with a relatively simple process, in which human samples are biotinylated and dialyzed in preparation for incubation, has become available using the largest available antibody array. The expression levels of 507 human target proteins, including angiogenic factors, cytokines and chemokines, can be analyzed simultaneously. A broad, panoramic view of cytokine expression can be obtained using this new technology.

Previously, we reported activated receptor tyrosine kinase (RTK) arrays in CRC (8). The downregulation of angiogenic factors such as vascular endothelial growth factor (VEGF) and matrix metalloproteinases inhibits invasiveness and tumor development in colon cancer (9). Since multistage carcinogenesis is observed during colon cancer development, it is suggested that colon cancer can be affected through multiple pathways. In the present study, we examined 507 human target proteins simultaneously using a new biotin label-based human antibody array in CRC samples.

\section{Materials and methods}

Patients. Tissue samples of CRC and normal colon tissues were obtained from 6 patients during surgery ( 2 males and 4 females; mean age, $71 \pm 11$ years; range, $56-92$ years). The clinicopathological data for the CRC patients are documented in Table I. Of these 6 patients, 1 had cancer localized to the ascending colon, 2 had cancer localized to the transverse colon, 1 had cancer localized to the sigmoid colon and 2 had 
Table I. Clinical characteristics of the colorectal cancer patients.

\begin{tabular}{lllcc}
\hline $\begin{array}{l}\text { Age } \\
\text { (years) }\end{array}$ & Gender & Region & Histology $^{\mathrm{a}}$ & $\begin{array}{c}\text { TNM } \\
\text { stage }^{\mathrm{b}}\end{array}$ \\
\hline 92 & Female & Ascending & WD & II \\
85 & Female & Transverse & MD & IIIa \\
63 & Male & Rectum & PD & IV \\
69 & Male & Sigmoid & WD & II \\
56 & Female & Transverse & MD & II \\
63 & Female & Rectum & WD & II \\
\hline
\end{tabular}

WD, well differentiated; MD, moderately differentiated; PD, poorly

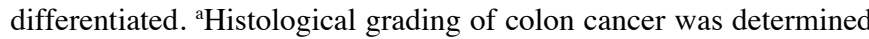
using criteria of the International Working Party. ${ }^{\text {bTNM stage was }}$ determined using the classification proposed by the International Union Against Cancer and the American Joint Committee on Cancer.

cancer localized to the rectum. Histologically, 3 cancers were well differentiated, 2 were moderately differentiated and 1 was poorly differentiated. With respect to clinical stage, 4 were in stage II, 1 was in stage IIIa and 1 was in stage IV. All experimental protocols were approved by the Human Subjects Committee of the Kagawa University School of Medicine. Informed consent was obtained prior to participation in the study.

Sample preparation. CRC tissue samples were prepared using methods previously described $(10,11)$.

Materials for the protein array. The RayBio ${ }^{\mathrm{TM}}$ Biotin Labelbased Human Antibody Array I (catalog no. AAH-BLM-1-2) was purchased from RayBiotech, Inc. (Norcross, GA, USA). This antibody array is a dot-blot based assay that enables the detection and comparison of 507 different human antibodies, including cytokines, chemokines, growth factors, angiogenic factors, proteases and soluble receptors.

Biotin label-based antibody array. This array was used according to the manufacturer's protocol. Homogenized tissues were dialyzed using a dialyzer. Biotin was added to the proteins that were derived from the colon tissues. Antibody array membranes were blocked for $1 \mathrm{~h}$ and incubated with $8 \mathrm{ml}$ of lysate from colon tissues at room temperature for $2 \mathrm{~h}$. After a washing step, the array membranes were incubated with HRP-conjugated streptavidin at room temperature for $2 \mathrm{~h}$. Unbound HRP-conjugated streptavidin was washed, and each array membrane was exposed to X-ray film using a chemiluminescence detection system (Amersham Life Sciences, Tokyo, Japan). A densitometric analysis was performed using ImageQuant TL (GE Healthcare Bioscience, Tokyo, Japan).

Data analysis. The array data of CRC and normal colon tissues were normalized using a positive control signal. The average local background signal was subtracted from the average signal intensity of duplicated spots for each antibody. A cluster analysis was performed to evaluate the associations among members of the groups or clusters.
Table II. Enhanced protein expression levels in colorectal cancer.

\begin{tabular}{lcc}
\hline & Ratio & P-value \\
\hline IL-1 $\alpha$ & 2.81 & 0.023 \\
GRO & 2.68 & 0.028 \\
Glut5 & 2.54 & 0.047 \\
MIG & 2.51 & 0.010 \\
ICAM5 & 2.29 & 0.046 \\
VE-cadherin & 2.28 & 0.026 \\
uPA & 2.26 & 0.017 \\
Leptin R & 2.13 & 0.045
\end{tabular}

IL-1, interleukin-1; GRO, growth-regulated oncogene; Glut5, glucose transporter 5; MIG, monokine induced by $\gamma$ interferon; ICAM5, intercellular adhesion molecule 5; VE-cadherin, vascular endothelial cadherin; uPA, urokinase plasminogen activator; Leptin R, leptin receptor.

Table III. Characteristics of the colorectal cancer patients.

No. No. of upregulated genes $(\mathrm{P}<0.01)$

\begin{tabular}{llc}
\hline $\begin{array}{l}\text { Gender } \\
\text { Male }\end{array}$ & 2 & 0 (in female vs. male) \\
Female & 4 & 0 (in $>65$ vs. $\leq 65)$ \\
Age (years) & & \\
$\leq 65$ & 3 & 0 (in MD/PD vs. WD) \\
$>65$ & 3 & \\
Histological grade & & \\
WD & 3 & 0 (in IIIa/IV vs. II) \\
MD/PD & 3 & \\
TNM stage & & \\
II & 4 & 23 (in rectum vs. non-rectum) \\
IIIa/IV & 2 & \\
Location & & \\
Non-rectum & 4 & \\
Rectum & 2 & \\
\hline
\end{tabular}

WD, well differentiated; MD, moderately differentiated; PD, poorly differentiated; TNM, tumor, regional lymph node, distant metastasis.

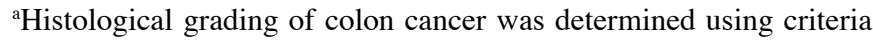
of the International Working Party. ${ }^{\mathrm{b}} \mathrm{TNM}$ stage was determined using the classification proposed by the International Union Against Cancer and the American Joint Committee on Cancer.

Statistical analysis. All analyses were conducted using the computer-assisted program JMP 8.0 (SAS Institute). A P-value of 0.05 represents a significant difference between groups.

\section{Results}

Enhanced expression of target proteins on biotin label-based antibody arrays. A total of 507 human proteins in CRC tissues 
A
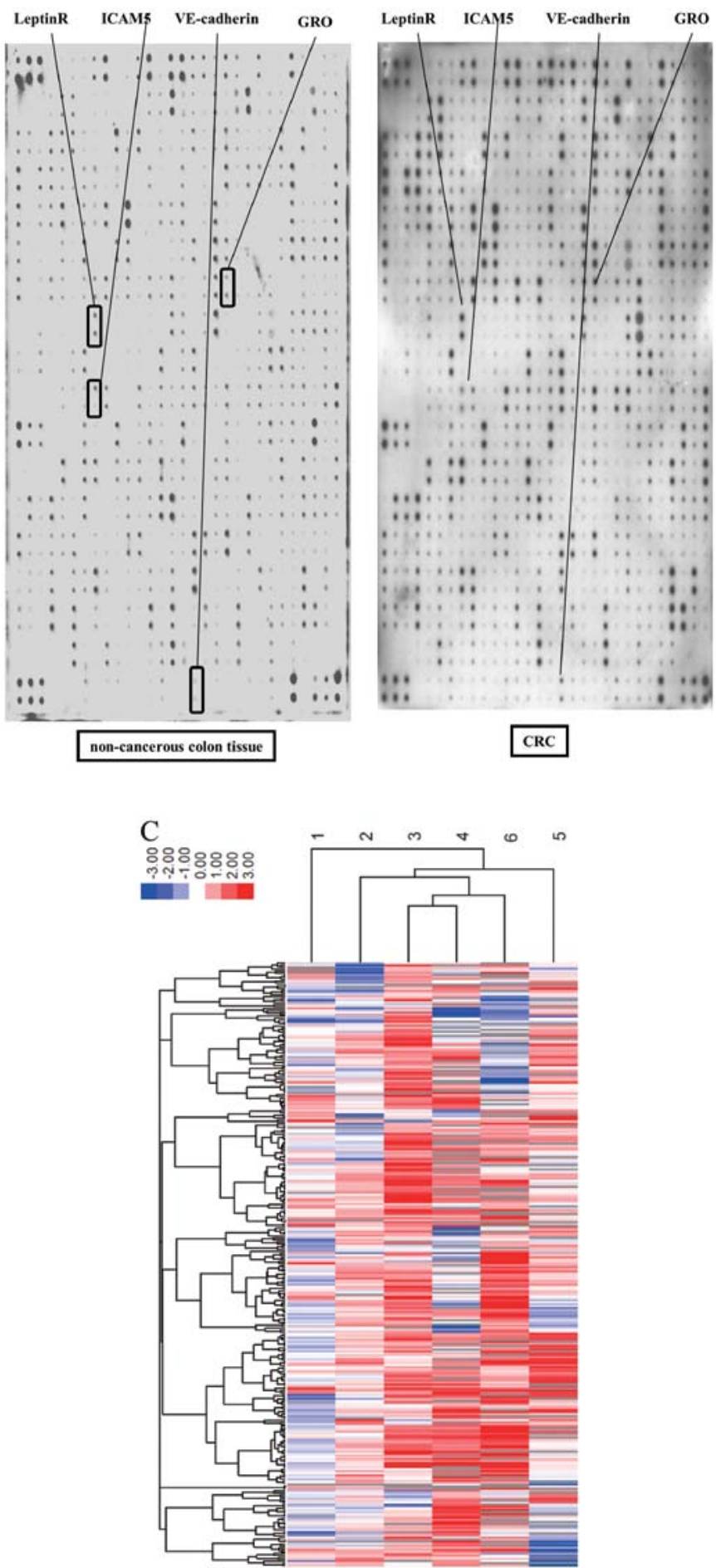

Figure 1. (A) Representative results of the biotin label-based antibody array. GRO, ICAM5, VE-cadherin and Leptin R were upregulated in colon cancer tissues as compared to normal colon tissue samples. (B) Representative results of the biotin label-based antibody array. IL-1 $\alpha$, Glut5, MIG and uPA were enhanced in colon cancer tissues as compared to normal colon tissue samples. (C) Hierarchical clustering of colorectal cancer and normal colon samples. Colorectal cancer samples were clustered according to the expression profile of 507 proteins. The protein clustering tree is shown at the top, and the sample clustering tree is shown to the left. The color scale represents the relative expression levels of target proteins; red represents a high expression level, and blue represents a low expression level.

were analyzed simultaneously on a single array. Representative antibody arrays between normal colon and CRC tissues are
B
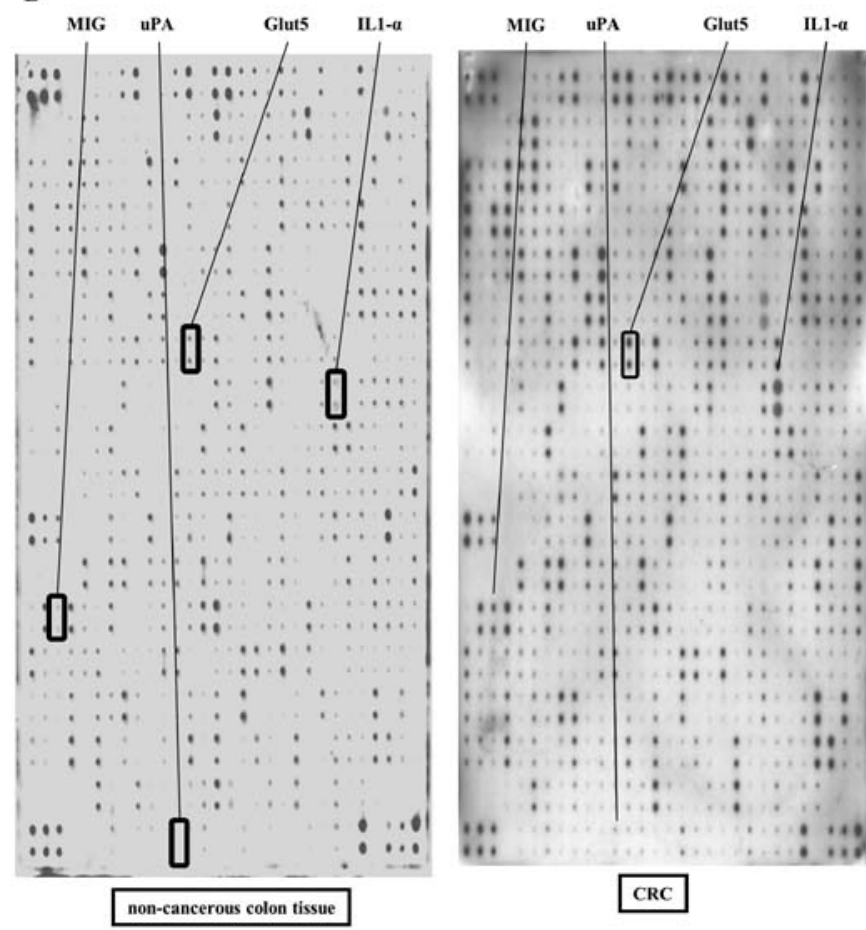

shown in Fig. 1A and B. IL-1 $\alpha$, GRO, Glut5, MIG, ICAM5, VE-cadherin, uPA and Leptin R were upregulated in CRC tissues when compared to these levels in normal colon tissues (Fig. 1A and B). A densitometric analysis was performed for each spot on the antibody array.

Unsupervised hierarchical clustering analysis using Pearson's correlation. To understand the results of the antibody protein array, unsupervised hierarchical clustering analysis was performed (Fig. 1C). IL-1 $\alpha$, GRO, Glut5, MIG, ICAM5, VE-cadherin, uPA and Leptin R were statistically significantly upregulated in CRC when compared to these levels in normal colon tissues $(\mathrm{P}<0.05)$ (Table II). No statistically significant protein downregulation was detected among the 507 human proteins on the array in CRC samples when compared to the normal colon tissues.

Significant upregulation of 23 target proteins in rectal cancer compared to non-rectal cancer. As shown in Table II, although no statistically significant protein upregulation was detected with respect to gender, age, histological grade or TNM stage, 23 target proteins were significantly upregulated in rectal cancer among CRC as compared to non-rectal cancer which consists of ascending, transverse and sigmoid colon $(\mathrm{P}<0.01)$ (Table III). Among these 23 target proteins, MPIF-1/CCL23, FGF R5 and MIP 2 were strongly upregulated in rectal cancer (Table IV).

\section{Discussion}

Various antibody protein arrays have been developed in the last decade. In most antibody array methods, two detection antibodies can recognize different epitopes of the same target protein and target antigens are visualized. However, an 
Table IV. Twenty-three target proteins in non-rectum (ascending, transverse and sigmoid colon)/rectum $(4 / 2)(\mathrm{P}<0.01)$ tissue samples.

\begin{tabular}{|c|c|}
\hline & P-value \\
\hline MPIF-1/CCL23 & 0.00035 \\
\hline FGF R5 & 0.00045 \\
\hline MIP 2 & 0.00072 \\
\hline SAA & 0.0019 \\
\hline IL-18 R $\beta$ (AcPL) & 0.00199 \\
\hline LFA-1 $\alpha$ & 0.00324 \\
\hline G-CSF R/CD 114 & 0.00328 \\
\hline IFN- $\gamma$ R1 & 0.00501 \\
\hline TRAIL R4/TNFRSF10D & 0.00507 \\
\hline FGF R3 & 0.0059 \\
\hline IL-18 R $\alpha$ (IL-1 R5) & 0.00613 \\
\hline GITR/TNFRF18 & 0.00658 \\
\hline IL-22 R & 0.00666 \\
\hline IL-18 BPa & 0.0075 \\
\hline E-Selectin & 0.00786 \\
\hline IL-17B & 0.00808 \\
\hline GDF1 & 0.00837 \\
\hline IL-1 F7/FIL1 $\zeta$ & 0.00839 \\
\hline ErbB4 & 0.00916 \\
\hline FAM3B & 0.00943 \\
\hline IL-27 & 0.00948 \\
\hline CRIM 1 & 0.00954 \\
\hline BMPR-II & 0.00955 \\
\hline
\end{tabular}

antibody cross-reaction can hamper accurate protein detection on the membrane of a protein array. To avoid this complication, surface plasmon resonance (SPR), which provides a label-free, single-antibody approach (12), has been developed. However, this approach also has additional complications, such as low detection sensitivity and a long waiting time to improve the instrumentation for high density detection. Therefore, in the present study, a biotin label-based human antibody array was used to perform the exhaustive analysis of protein expression. This technology enabled the detection of 507 proteins simultaneously, and a broad, panoramic view of target protein expression could be analyzed for each sample. Huang et al (13) reported that a biotin label-based antibody array could be used to profile the expression levels of many proteins simultaneously in ovarian cancer. These include cytokines, chemokines, adipokines, growth factors, angiogenic factors, proteases, soluble receptors and soluble adhesion molecules. Their finding supports the use of a biotin label-based antibody array targeted for 507 proteins in the present study.

In the present study, IL-1 $\alpha$, GRO, Glut, MIG and uPA were upregulated in colon cancer. These proteins have previously been reported as an angiogenic factor (14), anti-apoptotic factor, prognostic marker $(15,16)$, chemokine $(17,18)$ or in colon cancer. The present study data also demonstrated that ICAM5, VE-cadherin and Leptin R were upregulated in colon cancer (Table II). ICAM5 and VE-cadherin are cell adhesion molecules that are related to the development of breast cancer, prostate cancer and head and neck carcinoma (19-21). This finding suggests that these proteins could also be targetable molecules for colon cancer.

The array results also demonstrated that 23 target proteins were enhanced in rectal cancer (Table III). However, none of these proteins was a match to the target proteins that were presented in Table II. Recently, the Cancer Genome Atlas Network reported that hypermethylation was more common in the right colon than in the rectum (22). These findings strongly support our data that protein expression patterns differ between rectal cancer and non-rectal cancer.

In conclusion, our findings demonstrated that IL-1 $\alpha, \mathrm{GRO}$, Glut5, MIG, ICAM-5, VE-cadherin, uPA and Leptin R were upregulated in colon cancer when compared to levels in normal colon tissues using a biotin label-based antibody array. These results could aid in the simultaneous analysis of various types of upregulated proteins and promising target proteins for molecular-targeted therapies. The simplicity and ease of using the biotin label-based antibody array suggests that this new array method may be a powerful tool for detecting expression levels of a wide range of proteins from each pathway that contributes to colon carcinogenesis and for identifying new therapies for CRC.

\section{References}

1. Parkin DM, Bray F, Ferlay J and Pisani P: Estimating the world cancer burden: Globocan 2000. Int J Cancer 94: 153-156, 2001.

2. Paik SS, Jang SM, Jang KS, Lee KH, Choi D and Jang SJ: Leptin expression correlates with favorable clinicopathologic phenotype and better prognosis in colorectal adenocarcinoma. Ann Surg Oncol 16: 297-303, 2009.

3. Calle EE, Rodriguez C, Walker-Thurmond $\mathrm{K}$ and Thun MJ: Overweight, obesity, and mortality from cancer in a prospectively studied cohort of U.S. adults. N Engl J Med 24: 1625-1638, 2003.

4. Devine RM and Dozois RR: Surgical management of locally advanced adenocarcinoma of the rectum. World J Surg 16: 486-489, 1992

5. Wang Y, Cummings B, Catton P, et al: Primary radical external beam radiotherapy of rectal adenocarcinoma: long term outcome of 271 patients. Radiother Oncol 77: 126-132, 2005.

6. Prados J, Melguizo C, Ortiz R, et al: Colon cancer therapy: recent developments in nanomedicine to improve the efficacy of conventional chemotherapeutic drugs. Anticancer Agents Med Chem: Mar 4, 2013 (Epub ahead of print).

7. Huang RP, Yang W, Yang D, Flowers L, Horowitz IR, Cao X and Huang R: The promise of cytokine antibody arrays in the drug discovery process. Expert Opin Ther Targets 9: 601-615, 2005.

8. Morishita A, Gong J, Nomura T, et al: The use of protein array to identify targetable receptor tyrosine kinases for treatment of human colon cancer. Int J Oncol 37: 829-835, 2010.

9. Auyeung KK, Law PC and Ko JK: Novel anti-angiogenic effects of formononetin in human colon cancer cells and tumor xenograft. Oncol Rep 28: 2188-2194, 2012.

10. Yoshida S, Masaki T, Feng H, et al: Enhanced expression of adaptor molecule p46 Shc in nuclei of hepatocellular carcinoma cells: study of LEC rats. Int J Oncol 25: 1089-1096, 2004.

11. Yukimasa S, Masaki T, Yoshida S, et al: Enhanced expression of p46 Shc in the nucleus and p52 She in the cytoplasm of human gastric cancer. Int J Oncol 26: 905-911, 2005.

12. Boozer C, Kim G, Cong S, Guan H and Londergan T: Looking towards label-free biomolecular interaction analysis in a highthroughput format: a review of new surface plasmon resonance technologies. Curr Opin Biotechnol 17: 400-405, 2006.

13. Huang R, Jiang W, Yang J, et al: A biotin label-based antibody array for high-content profiling of protein expression. Cancer Genomics Proteomics 7: 129-141, 2010. 
14. Matsuo Y, Sawai H, Ma J, et al: IL-1 $\alpha$ secreted by colon cancer cells enhances angiogenesis: the relationship between IL-1a release and tumor cells' potential for liver metastasis. J Surg Oncol 99: 361-367, 2009.

15. Haber RS, Rathan A, Weiser KR, et al: GLUT1 glucose transporter expression in colorectal carcinoma: a marker for poor prognosis. Cancer 83: 34-40, 1998.

16. Langenskiold M, Holmdahl L, Angenete E, Falk P, Nordgren S and Ivarsson ML: Differential prognostic impact of UPA and PAI-1 in colon and rectal cancer. Tumour Biol 30: 210-220, 2009.

17. Ruehlmann JM, Xiang R, Niethammer AG, et al: MIG (CXCL9) chemokine gene therapy combines with antibody-cytokine fusion protein to suppress growth and dissemination of murine colon carcinoma. Cancer Res 61: 8498-8503, 2001.

18. Wen Y, Giardina SF, Hamming D, et al: GRO $\alpha$ is highly expressed in adenocarcinoma of the colon and down-regulates fibulin-1. Clin Cancer Res 12: 5951-5959, 2006.
19. Maruya SI, Myers JN, Weber RS, Rosenthal DI, Lotan R and El-Naggar AK: ICAM-5 (telencephalin) gene expression in head and neck squamous carcinoma tumorigenesis and perineural invasion! Oral Oncol 41: 580-588, 2005.

20. Sulkowska M, Famulski W, Wincewicz A, et al: Levels of VE-cadherin increase independently of VEGF in preoperative sera of patients with colorectal cancer. Tumori 92: 67-71, 2006.

21. Uddin S, Bavi PP, Hussain AR, et al: Leptin receptor expression in Middle Eastern colorectal cancer and its potential clinical implication. Carcinogenesis 30: 1832-1840, 2009.

22. Cancer Genome Atlas Network: Comprehensive molecular characterization of human colon and rectal cancer. Nature 487: 330-337, 2012. 